\title{
Analysis of Scattering by Inhomogeneous Dielectric Objects Using Higher-Order Hierarchical MoM
}

Kim, Oleksiy S.; Jørgensen, Erik; Meincke, Peter; Breinbjerg, Olav

Published in:

IV International Conference on Antenna Theory and Techniques

Publication date:

2003

Document Version

Publisher's PDF, also known as Version of record

Link back to DTU Orbit

Citation (APA):

Kim, O. S., Jørgensen, E., Meincke, P., \& Breinbjerg, O. (2003). Analysis of Scattering by Inhomogeneous

Dielectric Objects Using Higher-Order Hierarchical MoM. In IV International Conference on Antenna Theory and Techniques (pp. 113-116). IEEE.

\section{General rights}

Copyright and moral rights for the publications made accessible in the public portal are retained by the authors and/or other copyright owners and it is a condition of accessing publications that users recognise and abide by the legal requirements associated with these rights.

- Users may download and print one copy of any publication from the public portal for the purpose of private study or research.

- You may not further distribute the material or use it for any profit-making activity or commercial gain

- You may freely distribute the URL identifying the publication in the public portal 


\title{
ANALYSIS OF SCATTERING BY INHOMOGENEOUS DIELECTRIC OBJECTS USING HIGHER-ORDER HIERARCHICAL MOM
}

\author{
Oleksiy S. Kim ${ }^{1}$, Erik Jørgensen ${ }^{2}$, Peter Meincke ', and Olav Breinbjerg ${ }^{1}$ \\ ' Ørsted-DTU, Electromagnetic Systems, Technical University of Denmark \\ Ørsteds Plads, Building 348, DK-2800 Kgs. Lyngby, Denmark \\ <osk@oersted.dtu.dk> \\ ${ }^{2}$ TICRA, Laederstraede 34, DK-1201 Copenhagen K, Denmark
}

\begin{abstract}
An efficient technique for the analysis of electromagnetic scattering by arbitrarily shaped inhomogeneous dielectric objects is presented. The technique is based on a higher-order method of moments (MoM) solution of the volume integral equation. This higher-order MoM solution comprises recently developed higher-order hierarchical Legendre basis functions for expansion of the electric flux density and a higher-order geometry modeling. An unstructured mesh composed by trilinear (8node) and/or curved (27-node) hexahedral elements is used to represent the dielectric object accurately. It is shown that the condition number of the resulting MoM matrix is reduced by several orders of magnitude in comparison to existing higher-order hierarchical basis functions and, consequently, an iterative solver can be applied even for high expansion orders. Numerical results demonstrate excellent agreement with the analytical solutions for the dielectric sphere, as well as with results obtained by other numerical methods.
\end{abstract}

Keywords: volume integral equation, electromagnetic scattering, higher-order hierarchical basis functions, method of moments, inhomogeneous dielectric objects.

\section{INTRODUCTION}

Traditionally, scattering and radiation problems involving dielectric materials have been solved with surface integral equation formulations or by differential equation solvers such as the finite element method (FEM). A direct method of moment (MoM) solution of volume integral equations (VIE) has remained impractical due to its excessive CPU and memory requirements. Thus, various modifications of MoM have been developed to accelerate computations and relax computational demands for solution of VIE problems, e.g., the Conjugate-Gradient algorithm combined with Fast Fourier Transform (CG-FFT) [1] and the Fast Multipole Methods (FMM/MLFMM) [2] Being relatively fast these methods also have disadvantages: the first requires rectangular discretization grid and the second is still memory consuming.

An alternative approach is to reduce the number of unknowns in MoM considerably by discretizing the integral equation with higher-order interpolatory or hierarchical basis functions. Hierarchical functions provide greater flexibility, enabling different expansion orders on different elements in the same mesh. The main bottleneck of existing hierarchical basis functions is the ill-conditioning of the MoM matrix which requires a direct solver to be employed [3]
This implies that the solution time of the MoM matrix is proportional to $N^{3}$ where $N$ is the number of unknowns, whereas it is proportional to $N^{2}$ for iterative solvers. Thus, the application of an iterative solution method is essential for any efficient MoM tool for large-scale electromagnetic problems involving inhomogeneous dielectric materials.

Recently, a new type of higher-order hierarchical basis functions was proposed in [4], where they were applied to surface integral equations. Based on appropriately modified Legendre polynomials, the new basis functions are near-orthogonal and therefore provide low condition numbers of the MoM matrix. In this paper, we apply the new higher-order hierarchical Legendre basis functions to volume integral equations. The properties of the newly developed higher-order MoM solution are compared to existing higher-order formulations. Numerical examples for scattering by dielectric spheres demonstrate an excellent agreement between results of our higher-order hierarchical MoM and the Mie series analytical solution. A very low condition number of the MoM matrix allows the use of an iterative matrix solver even for high expansion orders. The memory requirements of the higher-order hierarchical MoM are compared with an existing multilevel fast multipole method (MLFMM) implementation [2]. 


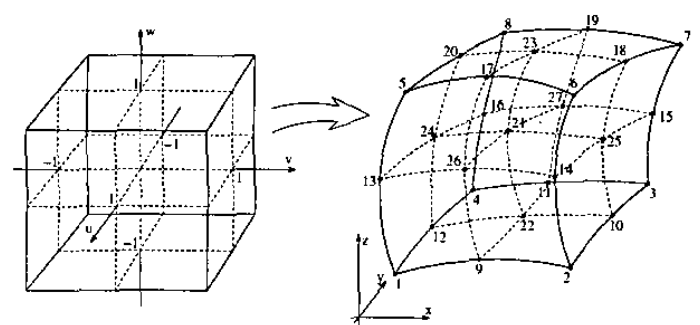

Fig. 1. Mapping between $(u, v, w)$ and $(x, y, z)$

\section{Higher-ORdER HiERARCHICAL MOM}

The discretization of an electric field volume integral equation with higher-order MoM involves two steps. First, a volumetric dielectric object is accurately represented by trilinear and/or curved hexahedral elements, which are defined by 8 and 27 nodes, respectively. Lagrange interpolation is employed to define an unique mapping between a cube in a curvilinear coordinate system $(u, v, w)$ and the hexahedron in the physical $(x, y, z)$ space, see Fig. 1. The dielectric permittivity inside each element is not necessarily constant but may vary as a function of coordinates.

Second, the unknown electric flux density $\mathbf{D}(u, v, w)$ in each local curvilinear $(u, v, w)$ coordinate system is expanded as

$$
\mathbf{D}(u, v, w)=\mathbf{a}_{u} D^{u}+\mathbf{a}_{v} D^{u}+\mathbf{a}_{w} D^{w},
$$

where $\mathbf{a}_{w}, \mathbf{a}_{v}$, and $\mathbf{a}_{w}$ are the covariant unitary vectors. Each of the contravariant components $\left(D^{u}, D^{v}, D^{u}\right)$ of $\mathbf{D}$ are expanded in terms of the higher-order hierarchical Legendre basis functions [4] as

$$
\begin{array}{r}
D^{\xi}=\frac{1}{A} \sum_{n=0}^{N^{n}} \sum_{q=0}^{Q^{\zeta}}\left\{C _ { 0 } ^ { \xi } \left[b_{0 n q}^{\xi}(1-\xi)+\right.\right. \\
\left.\left.+b_{1 n q}^{\xi}(1+\xi)\right] C_{n}^{\eta} P_{n}(\eta) C_{q}^{\zeta} P_{q}(\zeta)\right\}+ \\
+\frac{1}{A} \sum_{m=2}^{M^{\xi}} \sum_{n=0}^{N^{n}} \sum_{q=0}^{Q^{\zeta}}\left\{b _ { m n q } ^ { \xi } C _ { m } ^ { \xi } \left[P_{m}(\xi)-\right.\right. \\
\left.\left.\quad-P_{m-2}(\xi)\right] C_{n}^{\eta} P_{n}(\eta) C_{q}^{\zeta} P_{q}(\zeta)\right\}
\end{array}
$$

where $(\xi, \eta, \zeta)$ are $(u, v, w),(v, w, u)$, or $(w, u, v)$. In (2), $A$ is the Jacobian of the parametric transformation, $P_{m}(\xi)$ are Legendre polynomials, and $b_{m m q}^{\xi}$ are unknown coefficients. $M^{\xi}, N^{\eta}$, and $Q^{\zeta}$ denote the expansion orders along the three parametric directions. The factors $C_{m}^{\xi}, C_{n}^{\eta}$, and $C_{q}^{\zeta}$ are chosen such that the Euclidean norm of each function is unity for a unit cubic element. The basis functions are divergence-conforming, i.e., they satisfy continuity of the normal component of the electric flux density flowing through a face shared by two adjacent elements. Despite the necessary modifications to enforce the continuity, the expansion (2) maintains almost perfect orthogonality of the basis functions.
Due to the hierarchical property of the basis functions the expansion order can be selected separately in each hexahedron depending on the electrical size of the element. This allows large smooth objects to be represented by rather large curvilinear hexahedra with high expansion orders while fine parts of the geometry are precisely modeled by small hexahedral elements with low expansion orders. In this way, the number of unknowns can be minimized as opposed to low-order discretization schemes (volume RWG, or rooftops) that require small elements throughout the mesh. Furthermore, independent selection of the expansion order along the direction of the electric flux density and along the transverse directions provides additional flexibility to the discretization technique. Consequently, even geometries represented by elements that are far from cubic in shape, for instance thin dielectric shells or antenna radomes, can be treated in an efficient manner without introducing unnecessary unknowns.

\section{Numerical RESUltS}

First, consider a homogeneous dielectric sphere with dielectric constant $\varepsilon_{r}=4.0$. The sphere is placed at the origin of a rectangular $x y z$-coordinate system and illuminated by an $x$-polarized plane wave propagating in the $z$-direction. The size of the sphere is $k_{0} a=2.0$ where $k_{0}$ is a propagation constant in free space and $a$ is the radius. The sphere is represented with 32 hexahedral elements, both trilinear and curved. The expansion order is varied from $M^{\xi}=2$ to $M^{\xi}=4$ and the resulting number of unknowns, root-mean-square (RMS) error, and condition number of the MoM matrix are listed in Table 1. Here, the RMS error is computed for the bistatic radar cross section (RCS) relative to an exact analytical solution (the Mie series expansion). The RMS error is defined as

$$
R M S=\sqrt{\frac{\sum_{i=1}^{N_{n}}\left|\sigma_{M o M}-\sigma_{M i e}\right|^{2}}{\sum_{i=1}^{N_{n}}\left|\sigma_{M i e}\right|^{2}}},
$$

where $N_{n}$ is the number of sampling points (observation angles), $\sigma_{M o M}$ and $\sigma_{M i e}$ are the MoM and the exact RCS, respectively. The results are obtained by sampling the bistatic RCS at 181 angles in the E- and H-planes. The convergence with the RMS error less than $1 \%$ was achieved with $M^{\xi}=3$. A further increase of the expansion order yields a small improvement which probably indicates that geometrical modelling errors are dominating for $M^{\xi}=4$.

In Table 1 the 2-norm condition numbers for our higher-order Legendre basis functions and the existing higher-order power basis functions [3] are compared. It is observed that the higher-order hierarchical Legendre basis functions presented here provide very wellconditioned MoM matrix system for all expansion or- 
Table 1. Results for the homogeneous sphere

\begin{tabular}{|c|c|c|c|c|}
\hline Exp. Orde & $M^{\xi}$ & 2 & 3 & 4 \\
\hline Unknown & & 816 & 2700 & 6336 \\
\hline RMS erro & & $3.3 \cdot 10^{-2}$ & $6.8 \cdot 10^{-3}$ & $4.2 \cdot 10^{-3}$ \\
\hline Cond. & This paper & 84 & 159 & 346 \\
\hline Number & {$[3]$} & 1583 & $1.23 \cdot 10^{5}$ & $2.33 \cdot 10^{7}$ \\
\hline
\end{tabular}

ders. However, the condition numbers for the power basis functions [3] increase rapidly, approximately two orders of magnitude for each expansion order.

In the second example, a spherical shell of radius $a=2 \lambda_{0}$ and thickness $0.2 \lambda_{0}$ is analyzed. The dielectric constant of the shell is $\varepsilon_{r}=2.75-0.3 j$. In contrast to the previous example, where the expansion order was fixed for all hexahedra in all directions, the expansion order is now changed depending on the electrical size of each element. Thus, the expansion orders are chosen to be $M^{\xi}=4$ for the components tangential to the shell surface and $M^{\xi}=2$ for the radial components. In total 96 curvilinear hexahedral elements are used to represent the geometry of the shell. The solution with 10752 unknowns requires 884 Mbytes of memory. The obtained bistatic RCS is plotted in Fig. 2. Excellent agreement with the corresponding exact result is observed.

For this example, the MLFMM analysis with firstorder basis functions [2] requires 24642 hexahedra. 73962 unknowns, and 2GBytes of memory to achieve the same accuracy. Thus, despite the higher computational complexity of MoM $\left(O\left(N^{2}\right)\right)$ in comparison to MLFMM $(O(N \log N))$, the large reduction in the number of unknowns provided by the higher-order technique results in a more efficient solution.

As an example of an inhomogeneous dielectric object, we consider the scattering by a two-layer dielectric sphere with the inner radius $a_{1}=0.8 \lambda_{0}$ and outer radius $a_{2}=1.0 \lambda_{0}$. The dielectric constant and conductivity are $\varepsilon_{r 1}=4.0, \sigma_{1}=0.1 \mathrm{~S} / \mathrm{m}$, and $\varepsilon_{\mathrm{r} 2}=2.0, \sigma_{2}=0.05 \mathrm{~S} / \mathrm{m}$ for the inner and outer layer, respectively. 56 hexahedral elements and maximum expansion order $M^{\xi}=4$ are used in the

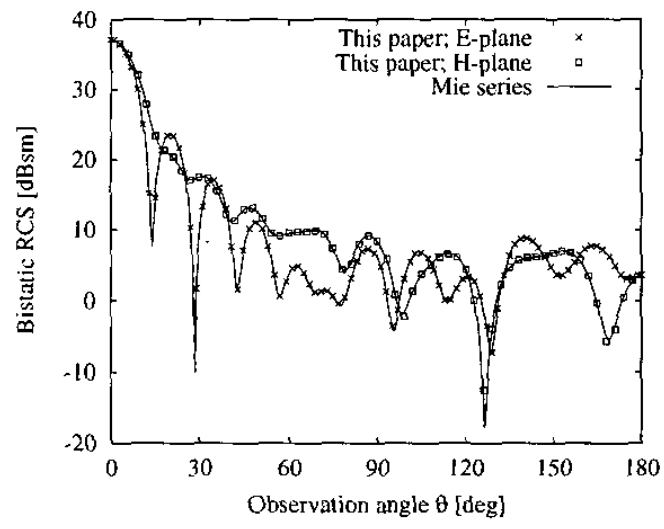

Fig. 2. Bistatic RCS for the spherical shell

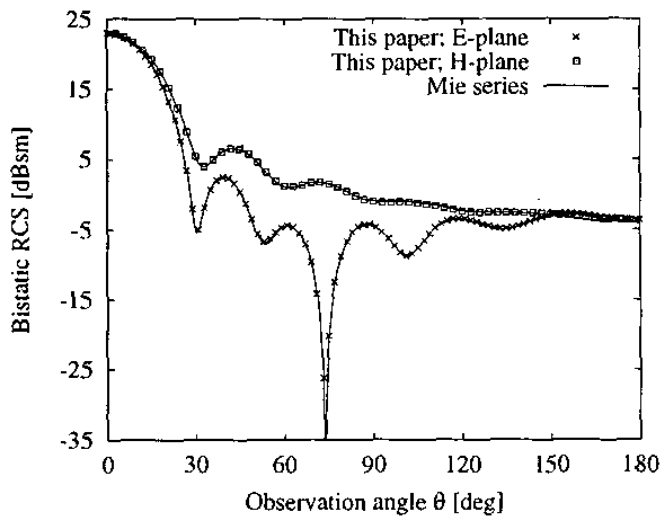

Fig. 3. Bistatic RCS for the two-layer sphere

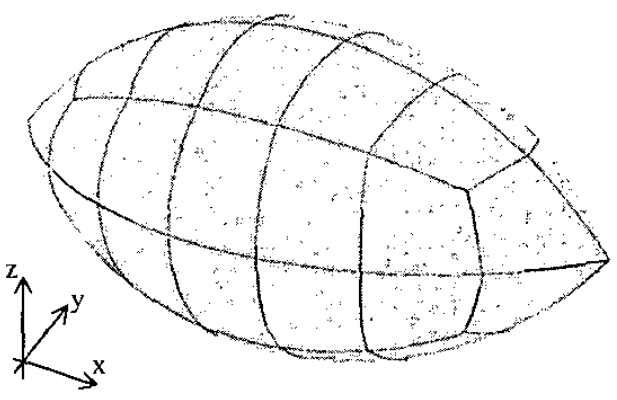

Fig. 4. Dielectric ogive

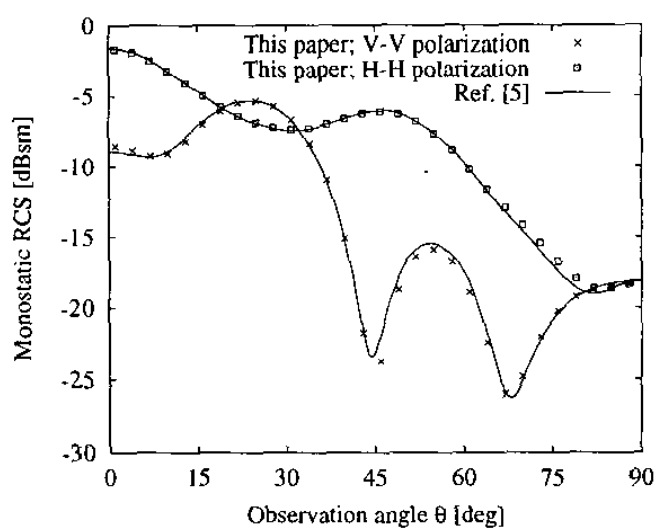

Fig. 5. Monostatic RCS for the dielectric ogive

solution. In Fig. 3, the bistatic RCS computed with 8640 unknowns at the frequency $f=300 \mathrm{MHz}$ is compared to the exact Mie series solution. Again, both results are in excellent agreement.

The scattering by a dielectric ogive (Fig. 4) is presented in the last example. The ogive is made of homogeneous dielectric with dielectric constant $\varepsilon_{r}=4.0-0.1 j$, and has a length $0.6 \mathrm{~m}$ and diameter $0.3 \mathrm{~m}$. The monostatic RCS computed in the $x z$-plane at the frequency $f=1 \mathrm{GHz}$ is shown in Fig. 5 . 
Our result obtained with 116 hexahedral elements, maximum expansion order $M^{\xi}=3$, and 6480 unknowns is in good agreement with the reference [5], where the surface integral equation technique is employed.

\section{Conclusion}

A new higher-order discretization scheme for volume integral equation is presented. The technique is based on higher-order hierarchical Legendre basis functions [4] for expansion of the electric flux density and higher-order geometry modeling. Improved orthogonality of the basis functions allows the condition number of the resulting MoM matrix to be reduced by several orders of magnitude in comparison to existing higher-order hierarchical basis functions and consequently, an iterative solver can be applied even for high expansion orders. Trilinear (8-node) and curved (27-node) hexahedral elements are used for accurate representation of dielectric objects. Numerical examples for scattering by a dielectric sphere and a shell demonstrate an excellent agreement between results of our higher-order hierarchical MoM and the Mie series analytical solution. It is shown that for fairly large scattering problems the higher-order hierarchical MoM requires mush fewer unknowns and less computer memory than low-order MLFMM.

\section{REFERENCES}

1. Catedra M. F., Gago E., Nuno L. A numerical scheme to obtain the RCS of three-dimensional bodies of resonant size using the conjugate gradient method and the fast Fourier transform // IEEE Trans. Ant. and Prop. 1989. Vol. AP-37. No 5, pp. 528-537.

2. Sertel K., Volakis J. L. Multilevel fast multipole method solution of volume integral equations using parametric geometry modeling // Proc. IEEE AP-S. 2001. Vol. 3, pp. 786-789. Boston, Massachusetts.

3. Notaros B. M., Popovic B. D., Weem J. P., Brown R. A., Popovic Z. Efficient large-domain MoM solutions to electrically large practical EM problems // IEEE Trans. Microw. Theory Techn. 2001. Vol. MTT-49. No 1, pp. 151-159.

4. Jørgensen E., Volakis J. L., Meincke P., Breinbjerg $\mathrm{O}$. Higher-order hierarchical Legendre basis functions for iterative integral equation solvers with curvilinear surface modeling // Proc. IEEE AP-S. 2002. Vol. 4, pp. 618-621. San Antonio, Texas.

5. Liu G., Gedney S. D. High-Order Moment Method Solution for the Scattering Analysis of Penetrable Bodies // Electromagnetics. 2003. Vol. 23. No 4, pp. 331-345, 2003. 